\title{
Introduction. L'évolution de la broderie de tradition byzantine en Méditerranée orientale et dans le monde slave (1200-1800)
}

\section{Elena Papastavrou et Marielle Martiniani-Reber}

\section{(2) OpenEdition}

\section{Journals}

Édition électronique

URL : https://journals.openedition.org/ceb/18264

DOI : $10.4000 /$ ceb. 18264

ISSN : 2261-4184

Éditeur

INALCO

Édition imprimée

ISBN : 9782858313983

ISSN : 0290-7402

Référence électronique

Elena Papastavrou et Marielle Martiniani-Reber, «Introduction. L'évolution de la broderie de tradition byzantine en Méditerranée orientale et dans le monde slave (1200-1800) », Cahiers balkaniques [En ligne], 48 | 2021, mis en ligne le 17 décembre 2021, consulté le 06 janvier 2022. URL : http:// journals.openedition.org/ceb/18264 ; DOI : https://doi.org/10.4000/ceb.18264

\section{(c) (1) (5)}

Cahiers balkaniques est mis à disposition selon les termes de la Licence Creative Commons Attribution - Pas d'Utilisation Commerciale 4.0 International. 


\title{
Introduction. L'évolution de la broderie de tradition byzantine en Méditerranée orientale et dans le monde slave (1200-1800)
}

\author{
Elena Papastavrou \\ Éphorat des Antiquités de Zakynthos \\ Marielle Martiniani-Reber \\ Musées d'art et d'histoire, Genève
}

Les broderies profanes ou liturgiques sont des témoins aussi bien artistiques, culturels et historiques, qu'économiques et politiques, ainsi que religieux et spirituels. Le présent volume est dédié à la broderie de tradition byzantine qui a évolué dans les pays héritiers de la culture byzantine, qui s'étendent à l'Est de la Méditerranée et au monde slave, entre 1200 et 1800. L'espace temporel a été ainsi délimité, car les broderies byzantines de caractère ecclésiastique, dont il est question principalement dans ce volume, existent depuis la fin du XII ${ }^{\mathrm{e}}$ siècle et que la tradition de cet art continue jusqu'à la révolution industrielle, ce qui pratiquement, dans le domaine artistique, apparaît après les premières décennies du XIX ${ }^{\mathrm{e}}$ siècle.

Jusqu'à ce jour, l'étude de la matière en question n'est pas aussi avancée que celles des autres disciplines, en raison d'une série de contraintes liées à l'approche de la matière. En effet, la broderie appartient à un art dont l'étude approfondie nécessite un équipement spécial (loupe, microscope, appareils et accessoires photographiques) qui peut davantage être fourni par une institution plutôt que par des chercheurs libres. De plus, les objets sont souvent placés dans des églises, des sacristies ou des dépôts à des endroits difficilement accessibles, dans des vitrines restées closes ou des placards cachés que prêtres et moines gardent jalousement. Ces contraintes mises à part, les publications dans des langues régionales dressent un autre obstacle qui interrompt la communication parmi les chercheurs de sorte qu'une grande part du matériel reste peu connue. 


\section{CAHIERS BALKANIQUES}

8 L'évolution de la broderie de tradition byzantine en Méditerranée orientale et dans le monde slave (1200-1800)

Notre ambition a été de rassembler dans ce volume les résultats de la recherche de spécialistes de différentes origines dans le but de contribuer essentiellement à la mise en lumière de l'évolution de la broderie de tradition byzantine d'un point de vue interdisciplinaire qui embrasse l'iconographie, le style et la technique, durant les périodes byzantine et post-byzantine.

Notre tâche est d'unir les forces des chercheurs pour que, petit à petit, se construise un langage commun qui ouvre des perspectives d'investigation : par exemple, comment les divers peuples héritiers de la culture byzantine ont-ils perçu la broderie byzantine ? Quel a été le rayonnement artistique de certains centres de broderie devenus importants après la chute de Byzance ? Enfin, quelles sont les influences orientales ou occidentales qui s'introduisent dans la broderie de tradition byzantine?

Dans cette optique, plusieurs thématiques s'ouvrent. Ainsi, en premier lieu, les deux premiers articles sont relatifs aux broderies byzantines des derniers siècles $\mathrm{du}$ Moyen Âge et à leur rapport avec les broderies occidentales tant par l'iconographie que par la technique. En effet, l'étude de Warren Woodfin, "Underside couching in the Byzantine World" [Le point couché retiré dans le monde byzantin], traite d'une série de broderies datées du XII ${ }^{\mathrm{e}}$ et du début du XIII ${ }^{\mathrm{e}}$ siècle, et originaires du nord de la Mer Noire. Leur caractéristique est liée au fait que les fils métalliques dorés sont brodés en couchés et retirés. Ceci est d'autant plus intéressant que la plupart des spécialistes considère que cette technique appartient exclusivement aux broderies occidentales. L'auteur démontre que, par opposition à ce que l'on pensait, la technique des fils couchés et retirés est présente également dans le monde byzantin. Ensuite, l'article de Marielle Martiniani-Reber, « Le parement d'autel de la Comtesse d'Étampes: une broderie réalisée dans le Duché d'Athènes ? » se réfère à une ouvre occidentale du $\mathrm{XIV}^{\mathrm{e}}$ siècle qui fait preuve d'influences byzantines tant iconographiques que techniques. Pour ces raisons, mais également en raison de l'historique de cette pièce, l'auteur propose sa production dans le Duché d'Athènes. C'est donc la première fois dans l'histoire de l'étude de la broderie de tradition byzantine qu'on est face à une œuvre mixte produite dans une région occupée par les Latins en Grèce.

En outre, l'article de Paschalis Androudis, «Quelques réflexions à propos de l'étoffe luxueuse du despote de Thessalonique, Andronic Paléologue, jadis conservée au monastère athonite de Vatopédi » porte notre attention sur une pièce brodée profane, dont aujourd'hui ne restent que des dessins complétés de descriptions non détaillées de l'étoffe par Fyodor Uspensky et la Mission Sevastianov. Il s'agit d'une contribution à l'iconographie de caractère héraldique dans les tissus byzantins du $\mathrm{Xv}^{\mathrm{e}}$ siècle. 
Encore, le travail d'Alice Isabella Sullivan, "Byzantine Artistic Traditions in Moldavian Church Embroideries" [Traditions artistiques byzantines dans les broderies moldaves à usage religieux] examine une série de broderies produites en Moldavie durant le $\mathrm{Xv}^{\mathrm{e}}$ siècle, après la chute de Constantinople. Tout en montrant les aspirations des commanditaires royaux, ces pièces, voiles liturgiques, vêtements sacerdotaux ou voiles funéraires, font preuve d'une influence importante de la tradition byzantine en iconographie, en style et en technique. Les analyses de l'auteur révèlent comment, à la fois, ces broderies préservent l'art de la capitale byzantine et évoluent, à la cour moldave, durant les années suivant 1453 .

Un secteur d'art religieux relativement inconnu est révélé par l'article de Nikolaos Vryzidis et d'Elena Papastavrou, "Notes on the Sanctuary Curtain : Symbolisms and Iconography in the Greek Church" [Voiles de «béma » orthodoxes grecs d'époque ottomane : symbolisme et iconographie]. Envisageant, d'abord, le symbolisme sacré attaché au «béma » et à son voile, l'étude se consacre, ensuite, d'une part aux voiles décorés de broderies de tradition byzantine et, d'autre part, aux voiles réalisés en tissus ottomans et safavides. Il s'agit, en effet, d'une contribution qui, entre autres, met en valeur un aspect de l'osmose entre la tradition byzantine et divers éléments de cultures orientales durant l'époque ottomane.

Par la suite, une série d'articles s'occupe de la période plus récente, qui va de la seconde moitié du XVII ${ }^{\mathrm{e}}$ siècle, jusqu'au début du XIX ${ }^{\mathrm{e}}$ siècle. Ainsi, le travail de Glycérie Chatzoulis, « À propos d'un épitrachilion de 1664 de la Métropole Trikkis et Stagon attribué à la broderie de l'école de Constantinople » enrichit nos connaissances sur la broderie à usage ecclésiastique orthodoxe-grecque issue des ateliers de la capitale ottomane. Ce travail est d'autant plus intéressant que les œuvres brodées orthodoxes grecques de provenance constantinopolitaine et portant une date antérieure à 1670 sont rares.

En outre, les divers courants liés à la broderie de tradition byzantine durant les $\mathrm{XVII}^{\mathrm{e}}$ et $\mathrm{XVIII}^{\mathrm{e}}$ siècles font l'objet de l'étude d'Elena Papastavrou et Daphni Filiou, "From Constantinople to Vienna: The different schools of Greek church embroidery $\left(17^{\text {th }}-18^{\text {th }}\right.$ centuries" [De Constantinople à Vienne : à propos de différentes écoles de broderie ecclésiastique grecque (XVII ${ }^{\mathrm{e}}-\mathrm{XVIII}^{\mathrm{e}}$ siècles)]. En fait, les ateliers de Constantinople, de Vienne et de Crète, aussi bien que ceux du Pont-Euxin et de Géorgie, sont étudiés sous une approche interdisciplinaire qui vise à l'approfondissement dans la méthode de construction technique des broderies en combinaison avec l'examen de l'iconographie et du style.

Par la suite, un thème original aussi bien qu'un témoignage historique sont offerts par une paire de manipules (épimanikia) que Nikos Mertzimekis traite dans son article : «Vêtements sacerdotaux russes brodés d'or au monastère d'Iviron 
du Mont Athos : source de savoir et de mémoire ». Outre l'iconographie unique fournie par leur décor brodé, ces vêtements sacerdotaux conservés au monastère d'Iviron n'attestent que des relations fraternelles en Christ entre les Russes et les Athonites durant les XVIII ${ }^{\mathrm{e}}$ et $\mathrm{XIX}^{\mathrm{e}}$ siècles.

Enfin, ce volume s'achève par une étude d'Anna Karatzanis, "The use of metal threads in the decoration of late and post Byzantine embroidered church textiles" [L'emploi des fils métalliques dans la décoration des tissus brodés byzantins et post-byzantins à usage religieux]. En fait, l'analyse de petits échantillons de tissus brodés se trouvant dans des Fondations grecques fournit des informations aussi bien sur les types de fils métalliques que sur les techniques et les matériaux de fabrication de broderies de tradition byzantine datées du XIV ${ }^{e}$ au XIX ${ }^{e}$ siècle.

Ainsi, dans l'espoir que ce volume, qui ne constitue que les prémisses de l'effort d'unir les travaux des spécialistes, a atteint une petite partie de nos ambitions premières, nous souhaiterions avoir l'occasion d'approfondir cette recherche dans le domaine de la broderie de tradition byzantine dans des futurs ouvrages. 